\title{
Prática Baseada em Evidências e a análise sociocultural na Atenção Primária
}

\author{
| ${ }^{1}$ Luana Roberta Schneider, ${ }^{2}$ Rui Pedro Gomes Pereira, ${ }^{3}$ Lucimare Ferraz |
}

Resumo: Prática Baseada em Evidências é uma abordagem que tem apresentado notoriedade para melhorar a efetividade clínica e apoiar o profissional de saúde nas suas condutas, utilizando três elementos: evidências científicas, a experiência clínica e as preferências do paciente. O objetivo do estudo é conhecer como se desenvolve a Prática Baseada em Evidências na Atenção Primária à Saúde, especificamente, na Estratégia Saúde da Família, bem como propor a inclusão do elemento "análise sociocultural" para a tomada de decisão. Estudo qualitativo, precedido da realização de dois grupos focais, com a participaçâo de 14 profissionais de saúde. A técnica de análise de conteúdo temática foi utilizada para avaliaçáo, tratamento e interpretaçáo das informaçóes. Os profissionais da Estratégia Saúde da Família têm dificuldade para desenvolver uma prática em saúde voltada a evidências científicas, apontando carência de conhecimentos e habilidades para a pesquisa e elevada carga de trabalho. Ademais, os resultados indicam que a Prática Baseada em Evidências deve ser diferenciada segundo a realidade dos territórios de atuação das equipes. Cabe aos profissionais de saúde identificar e valorar as características socioculturais da comunidade para uma Prática Baseada em Evidências mais sensível às necessidades de saúde de sua população adscrita.

> Palavras-chave: Prática Baseada em Evidências; Atenção Primária à Saúde; Estratégia de Saúde da Família; características culturais.

\author{
1 Doutorado, Programa de Pós- \\ Graduação em Ciências da Saúde, \\ Universidade Comunitária da \\ Região de Chapecó. Chapecó- \\ SC, Brasil (luanaschneider@ \\ unochapeco.edu.br). \\ ORCID: 0000-0001-9724-8667 \\ 2 Universidade do Minho, \\ Braga, Portugal (ruipereira@ese. \\ uminho.pt). \\ ORCID: 0000-0002-4811-6753 \\ ${ }^{3}$ Programa de Pós-Graduação em \\ Ciências da Saúde, Universidade \\ Comunitária da Região de \\ Chapecó. Chapecó-SC, Brasil \\ (Iferraz@unochapeco.edu.br). \\ ORCID: 0000-0002-2487-8614
}

Recebido em: 04/08/2019 Aprovado em: 03/03/2020 Revisado em: 30/06/2020 


\section{Introdução}

A Prática Baseada em Evidências (PBE) tem sido considerada uma importante mudança de paradigma na educação e no âmbito da saúde moderna (EBELL et al., 2017). Ela é definida como uma abordagem que associa a melhor evidência científica disponível, com a experiência clínica e a escolha do paciente para auxiliar na tomada de decisão (SACKETT et al., 2003). O termo "baseado em evidências" foi originado nos anos 1990 proveniente da medicina, embora hoje seus princípios se estendam por disciplinas tão variadas quanto enfermagem, odontologia, educação, serviço social e políticas públicas. Seu uso, pelos profissionais da saúde, configura-se como uma forma coerente, segura e sistematizada para prover maior qualidade na assistência e a otimização dos recursos, alcançando a eficácia e a relaçáo custo-benefício da prestação de cuidados em saúde (SAUNDERS; VEHVILAINEN-JULKUNEN, 2017; PEREIRA; CARDOSO; MARTINS, 2012; SACKETT et al., 2003).

Ainda assim, várias têm sido as barreiras que dificultam a implementação desta abordagem, dentre as quais se destacam: ausência de conhecimento sobre PBE e habilidades para sua aplicação, falta de crença que a PBE possibilite resultados mais positivos comparados ao cuidado tradicional, quantidade volumosa de informaçôes nos periódicos, falta de tempo e recursos para buscar e avaliar as evidências disponíveis, carência de apoio administrativo ou incentivo das instituiçóes e resistência para mudanças (SHAFIEI et al., 2014; AMMOURI et al., 2014; PEREIRA, 2016; ZHOU et al., 2016; SHIN; LEE, 2017).

A utilização da PBE permite diminuir as distâncias entre a pesquisa e a prática assistencial, pois sua implementação ocorre por meio da avaliação dos resultados obtidos das pesquisas, a partir da busca e avaliação crítica das evidências. Contudo, há críticas de suas limitaçôes como um padrão único para a prática, baseado em que a produção de evidências não ocorre em igualdade de condiçóes. Por mais bem intencionadas que sejam, se as evidências estão construídas a partir de uma epidemiologia convencional, que segundo Breilh (2015, p. 535) “[...] não integra processos nas múltiplas dimensôes e não relaciona as questôes com a estrutura socioeconômica, política, e com os condicionamentos culturais, elas serão obviamente focalizantes, e, evidentemente, as açôes serão igualmente reducionistas".

A PBE estrutura a conduta, mas não substitui o julgamento clínico e a experiência pessoal. Considera-se que sem a experiência prática, a evidência, mesmo 
sendo considerada de excelência, poderá ser inadequada em determinada situação individual. Por outro lado, sem recorrer à melhor evidência disponível, há o risco de manter práticas desatualizadas, com os inerentes prejuízos para as pessoas (SACKETT et al., 1996). Isso significa que os profissionais de saúde deveriam utilizar tanto suas vivências profissionais, quanto as melhores evidências disponíveis na sua tomada de decisão clínica, pois uma evidência científica de qualidade pode não se aplicar a determinado paciente.

No campo da Atenção Primária à Saúde (APS), especificadamente, na Estratégia Saúde da Família (ESF) há uma lacuna de estudos sobre a incorporação da PBE. Este é considerado um cenário de atuação complexo devido ao amplo leque de atividades e competências dos profissionais e o papel da equipe como organizadores da demanda local e das ações de saúde do seu território. Ressalta-se que esses territórios não são homogêneos e configuram-se num espaço social, com determinada realidade de saúde da população que nele vive e que está em constante movimento, com suas dimensôes políticas, econômicas, epidemiológicas e culturais (TAKEDA, 2013).

Considerando a complexidade do trabalho da ESF, entende-se que no campo da APS a PBE precisa avançar. Portanto, diante dessa realidade, o presente estudo objetivou conhecer como os profissionais da equipe da ESF desenvolvem a Prática Baseada em Evidências em um município do Sul do Brasil e propor a inclusão do elemento "análise sociocultural" para a tomada de decisão, dos profissionais de saúde, frente às demandas da comunidade.

\section{Metodologia}

Trata-se de pesquisa descritiva, de abordagem qualitativa, realizada no município de Chapecó, situado na região oeste do estado de Santa Catarina, Brasil, com população de aproximadamente 210 mil habitantes (IBGE, 2010). O cenário de estudo contemplou dois Centros de Saúde da Família com equipes de Estratégia Saúde da Família, selecionados, intencionalmente, pelos pesquisadores, que comportavam, no mínimo, profissionais médicos, enfermeiros e cirurgiōes-dentistas.

As informaçôes foram coletadas por meio da realização de dois grupos focais, um em cada centro de saúde. Para tanto, foi realizado um contato telefônico, para as coordenadoras dos dois Centros de Saúde da Família, no intuito de explicar o objetivo da pesquisa e agendar a data e o horário de acordo com disponibilidade 
de cada equipe. Os grupos foram realizados no local de trabalho dos envolvidos. No primeiro grupo participaram dois médicos, dois enfermeiros e um cirurgiãodentista. No segundo participaram três médicos, quatro enfermeiros e dois dentistas, totalizando 14 trabalhadores ao final dos dois grupos focais. Cada profissional, a fim de preservar sua identidade, escolheu um codinome (espécie de flor) e utilizou no formato de um crachá. O roteiro norteador apresentado ao grupo continha a seguinte questão de partida para o debate: o que vocês entendem por PBE? Depois emergiram outras, a saber: quais as dificuldades/entraves para desenvolverem PBE? Como a PBE é realizada/percebida no serviço de saúde?

Essa técnica possibilitou extrair mais facilmente a expressão dos participantes, visto que, no processo de interação, os comentários estimularam que todos os participantes se expressassem e, ao final, consensuaram suas opinióes. Todo diálogo foi gravado em dois gravadores digitais para garantir a totalidade das falas e o tempo de realização dos grupos variou de 40 minutos até uma hora.

Para a análise, as informações foram transcritas e organizadas com auxílio dos softwares Excel e Word, permitindo uma leitura panorâmica do conteúdo. Posteriormente, foram analisadas empregando a técnica de análise de conteúdo temática, que consistiu na identificação dos núcleos de sentido a partir da presença ou da frequência do tema que compôe o texto, desde que houvesse relação com os objetivos da pesquisa. A análise de conteúdo temática teve como base a sequência de etapas proposta por Bardin (2016): pré-análise, exploraçáo do material e tratamento dos resultados obtidos e interpretação. Da análise emergiram duas categorias que foram predefinidas de acordo com os objetivos do estudo: 1) A Prática Baseada em Evidência na Atençáo Primária à Saúde; e 2) A proposta de um novo elemento da Prática Baseada em Evidências na Atenção Primária à Saúde: análise sociocultural. Nessa investigação foram seguidos os procedimentos éticos recomendados, obtendose a aprovação de Comitê de Ética em Pesquisa sob o parecer de número 1.573.371.

\section{Resultados e Discussão}

As categorias identificadas trouxeram à tona os elementos que interferem no desenvolvimento pleno da PBE na APS. Ademais, os resultados indicam que a PBE deveria ser diferenciada para todos os territórios que a equipe atua, reconhecendo a realidade, suas necessidades e diferenças. 


\section{A PBE na APS}

Os profissionais de saúde que atuam na APS valoram a experiência clínica em suas práticas em saúde. Observou-se, ainda, que essa experiência clínica não é apenas a pessoal, mas também aquela que o profissional adquire com o colega, quando socializa suas dúvidas, ou seja, quando busca e compartilha a base do conhecimento empírico entre a equipe de saúde. A experiência pessoal foi obtida como o suporte inicial para os trabalhadores, assim como estes se sentiriam mais confiantes se houvesse uma pessoa com conhecimento em investigação para ajudar e sintetizar os resultados das pesquisas (PEREIRA, 2016, YODER et al., 2014).

Vivência profissional, do conhecimento que a gente vai adquirindo (Girassol).

Baseada em experiência em que outros profissionais tiveram (Amor perfeito).

Eu nunca tive acesso ao UpToDate [base de informaçóes médicas baseadas em evidências] você busca um colega, junto com esse colega você busca a experiência que a equipe tem, e as vezes, quando náo se resolve ainda, ligamos para algum outro profissional (Onze horas).

Cada um tem o seu saber. Então a gente tem que somar (Girassol).

A gente chama o colega. O colega vem, avalia e troca informaçóes com um terceiro colega, às vezes (Rosa).

Os resultados também permitiram compreender que os profissionais apresentam dificuldades para operacionalizar as etapas da PBE, como converter a necessidade de cuidado identificada em uma pergunta, realizar a busca na literatura, interpretar e aplicar as evidências científicas disponíveis (SADEGHI-BAZARGAN; TABRIZI; AZAMI-AGHDASH, 2014, MALLION; BROOKE, 2016, SHIN; LEE, 2017). Os obstáculos para a busca de evidências podem ser identificados nas seguintes falas:

Eu tenho essa dificuldade [de acessar bancos de dados] a gente tem que se virar na internet, eu perco muito tempo procurando informação (Onze horas).

$\mathrm{Na}$ área enfermagem a gente tem dificuldade com a questão das evidências [..] (Margarida).

São várias as razóes pelas quais os profissionais, em muitas áreas da prática, não consultam evidências científicas. Atitudes individuais desfavoráveis e normas sociais adotadas por seus pares muitas vezes desencorajam os praticantes de adotarem práticas baseadas em evidências científicas. As restriçóes individuais do profissional também tendem a limitar o uso da PBE devido às barreiras percebidas em seus ambientes de trabalho (BARENDS et al., 2017). 
A carência de conhecimentos e habilidades, que muitas vezes não é solucionada durante a formação do profissional, pode impactar nos resultados positivos de saúde da população. Estudo realizado com o objetivo de determinar até que ponto as recomendaçôes, para a prática de cuidados primários, são informadas por evidências baseadas em pesquisas científicas demostrou que apenas 18\% são alicerçadas em evidências orientadas para o paciente a partir de estudos de alta qualidade (EBELL et al., 2017). Entende-se que realizar uma prática baseada em evidências, de forma integral, requer dos profissionais da saúde uma capacitação para o desenvolvimento de estratégias que auxiliem a utilização de pesquisas no cotidiano, a fim de transpor a dicotomia entre teoria e prática (CAMARGO et al., 2016).

Ademais, percebeu-se que essa carência de conhecimentos e habilidades em fazer busca de artigos, manusear os bancos de dados, selecionar as informaçóes muitas vezes advém de sua formação. Segundo os profissionais, isso não foi exercitado durante o curso na área da saúde, revelando uma deficiência na graduação quanto à abordagem da PBE. Estudo realizado na Bahia revelou que a maioria dos formandos do curso de graduação em Enfermagem considera haver déficit de metodologias de ensino voltadas para o incentivo à iniciação científica e a produção de pesquisas (SANTOS; ANJOS; ALMEIDA, 2013).

\footnotetext{
Existe uma grande dificuldade, acho que a universidade tem muito para fazer ainda. Falta muito na formação [Prática Baseada em Evidência] (Amor-perfeito).

Eu fiz apenas uma aula de pesquisa e medicina baseada em evidência, e foi bem legal, pela primeira vez na minha vida assistir uma aula técnica assim, mas daí, não fiz mais nada (Amor-perfeito).
}

Outrossim, alguns participantes se formaram há muito tempo, quando essa prática não era comumente conhecida e referidas nas disciplinas. O conceito da PBE é relativamente moderno e originou-se a partir da Medicina Baseada em Evidências, organizada no início dos anos 80, na Universidade McMaster, no Canadá (SACKETT et al., 1996).

A minha formação é bem antiga. Não se falava, não se discutia na graduação [a busca por evidências científicas] (Hortência).

A falta de formação e, consequentemente, o déficit de conhecimentos e habilidades, para a busca de evidências científicas, faz com que os profissionais demandem muito tempo para se atualizem. Contudo, relataram que outro aspecto 
que limita a realização da PBE é a elevada carga de trabalho e que falta uma estrutura organizacional que favoreça o desenvolvimento dessa abordagem.

A gente tem que fazer mais pelo paciente, mas não dá tempo, porque é muito coisa, muito paciente, muita demanda (Amor perfeito).

A agenda está sempre cheia (Lírio).

A internet é meio lenta, tem inclusive os filtros que eles colocam lá na prefeitura, pro pessoal não acessar, e as vezes fica um pouco difícil [fazer buscas de evidências] (Onze-horas).

Os profissionais, em geral, percebem a importância da PBE e aceitam a necessidade de implementá-la, mas a falta de tempo no trabalho pode desestimular seu uso (ZHOU et al., 2016). A alta demanda de atividades é um entrave reportado em diversos estudos equivalentes (NAVABI et al., 2014; PERICAS-BELTRAN et al., 2014; MORALES; SANTACRUZ; VERKOVITCH, 2015; MALLION; BROOKE, 2016). Por essa razão, em disciplinas onde a PBE está bem estabelecida, evidências pré-avaliadas na forma de revisóes sistemáticas, síntese de evidências ou outros tipos de resumos são fornecidos por comunidades globais, a exemplo da Cochrane. Esses resumos permitem que os profissionais consultem rapidamente as melhores evidências científicas disponíveis sobre questôes de interesse, verifique a literatura de pesquisa para novas descobertas, e atualizem seus conhecimentos profissionais à medida que surgem novas demandas (COCHRANE BRASIL, 2019).

Embora não seja suficiente para mudar a prática, uma cultura de aceitação e apoio de gestores e colegas pode ser considerada como elemento facilitador para o fortalecimento da PBE nos serviços de saúde (BAIRD; MILLER, 2015). Compreendese que os profissionais tenham maior probabilidade de incorporar evidências de pesquisa em suas práticas quando formarem atitudes positivas em relação à pesquisa científica, estiverem expostos a normas sociais de apoio em relação a seu uso e virem as barreiras para seu uso como superáveis (BARENDS et al., 2017).

Diante dessas dificuldades - conhecimentos, habilidades e acesso - o uso de protocolos disponibilizados pelo Ministério da Saúde, do Brasil, são ferramentas, amplamente, utilizadas pelos profissionais na sua rotina de trabalho na APS. Estudo de Pereira, Cardoso e Martins (2012) com 95 enfermeiros, em Portugal, indicou que os protocolos foram as fontes de informaçóes mais utilizadas para aquele grupo investigado. Os protocolos são considerados ferramentas essenciais na organização do processo de trabalho e na resolubilidade das açôes de saúde, 
mas orienta-se que sejam usados numa lógica de normalização e nunca de uniformização de práticas e/ou cuidados de atenção à saúde (AGREE, 2009; WERNECK; FARIA; CAMPOS, 2009).

Eu pelo menos sempre costumo seguir os protocolos estabelecidos pelo Ministério da Saúde, o que está sendo feito na atualidade é procurar seguir protocolos (Tulipa).

Eu utilizo o protocolo do ministério [da saúde], procuro e já deixo baixado no computador (Violeta).

Vou no Ministério da Saúde pra ver o que aparece, quando não encontro as vezes entro no site de Cuba que eu já conheço (Onze horas).

Eu vou mais pra área do ministério da saúde mesmo (Rosa).

Em síntese, as instituiçóes de ensino poderiam se concentrar em melhorar as habilidades de PBE necessárias para entender, pesquisar e aplicar evidências científicas. Por outro lado, é necessário maior esforço por parte dos pesquisadores para resumir/traduzir a pesquisa de forma amigável aos profissionais que atuam na prática (BARENDS et al., 2017).

\section{A proposta de um novo elemento da PBE na APS: análise sociocultural}

A respeito dessa categoria, não foram identificadas experiências nacionais com caráter similar, o que revela o aspecto inovador desta produção e potencial para contribuir com a implementação da PBE no âmbito da APS.

A aplicação de evidências de pesquisa na prática envolve consideraçóes complementares acerca do contexto. Os precursores da PBE reconhecem a necessidade de aliar, com atenção, as evidências científicas aos valores e os diferentes cenários de vida dos pacientes. A pesquisa científica envolve relaçôes entre teoria, método e problema científico - esse tripé pode entrecruzar outras formas de conhecimento que provém das crenças, do senso comum e da religiáo. No entanto, o processo de integração de múltiplas formas de conhecimento na prática clínica permanece pouco estudado (KIRMAYER, 2012).

A partir dos grupos focais, além da atitude dos profissionais em buscar informaçóes científicas aliando a sua experiência e a preferência do paciente, a análise sociocultural surge como um elemento central para contemplar as informaçóes dos territórios/ comunidades onde a equipe atua. O contexto de trabalho dos profissionais da APS é variado. Assim, é relevante adaptar a evidência científica que está disponível, no local de trabalho, visto que cada ambiente apresentará diferentes circunstâncias para que 
a evidência seja realmente colocada em prática (BANDEIRA et al., 2017). Condutas baseadas em dados da maioria da população podem ter uma relevância incerta para grupos culturais específicos. Desta forma, é recomendável desenvolver estratégias especializadas, bem como fornecer os recursos necessários para a implementação de práticas baseadas em evidências devidamente adaptadas ao campo da APS (PERICASBELTRAN et al., 2014). Os depoimentos a seguir, exemplificam os resultados:

Tem que ir desenvolvendo as próprias experiências de acordo com a nossa região, de acordo com a nossa cultura, de acordo com a situação que vai se apresentar [...]. A saúde da própria comunidade, da nossa localidade (Onze horas).

A comunidade aqui tem muita necessidade, tem outro nível intelectual então obviamente a problemática dos pacientes aqui não é igual em outro bairro (Lírio).

A evidência é por bairro, existe muita diferença entre cada unidade de saúde e muita diferença de áreas dentro da unidade de saúde, [...], as doenças se equiparam, mas o nível sociocultural é diferente, entáo o próprio bairro, as áreas e micro áreas são diferentes (Jasmim).

Os aspectos socioculturais são compostos pelo ambiente, cultura, economia, dados demográficos, crenças e valores (BREILH, 2015). Deste modo, o contato dos profissionais da APS junto à comunidade resulta não apenas em coordenar o cuidado de pessoas que constroem relações entre si, mas também permite observar os fenômenos humanos e contradiçôes sociais que ocorrem nesse meio (SPERLING, 2017).

Os valores e preferências do paciente incluem as expectativas únicas que cada usuário e membro da família traz para o atendimento com o profissional de saúde. As questóes culturais são cruciais para todos os cuidados clínicos, aconselhamento preventivo, diagnóstico, tratamento e tratamento da doença, porque a cultura molda as crenças, os valores e, portanto, os comportamentos relacionados à saúde. O encontro clínico é fundamentado na comunicação, onde os valores e crenças do paciente e do clínico sáo compartilhados. Quando o foco dos cuidados de saúde está na doença sem um contexto, há uma distorção da realidade clínica (MONTENERY et al., 2013).

Muito além de ser simplesmente o espaço operativo do sistema de saúde, é no território onde se verifica a interação dos indivíduos/comunidade com os serviços locais (MONKEN; BARCELLOS, 2005). Estar atento aos modos de organização e de sobrevivência das pessoas no seu cotidiano possibilita aos profissionais da saúde 
uma visão não fragmentada dos diversos processos envolvidos (BRASIL, 2017). De maneira convergente, considerar os dados epidemiológicos do território, bem como as crenças e valores da população, justifica-se pelo fato de que o ambiente exerce influência decisiva nas manifestaçôes das doenças, na busca de tratamento e na relação que os usuários estabelecem com os serviços de saúde, sendo que para melhor intervir sobre os comportamentos individuais e coletivos, é essencial, particularmente pelos profissionais que atuam na APS, conhecer o contexto sociocultural no qual estão inseridos (BREILH, 2015).

Tem havido crescente conscientização da necessidade de incluir os profissionais e a comunidade nos processos de pesquisa. Essa abordagem de pesquisa colaborativa e orientada para a comunidade são vistas como uma forma de desenvolver pesquisas que abordam as lacunas da prática e refletem as necessidades e prioridades das pessoas que são as mais afetadas pelos resultados da pesquisa (BANNER et al., 2019). Esse envolvimento e diálogo entre pesquisadores, profissionais e a comunidade é considerada uma estratégia de pesquisa altamente inclusiva, que favorece o aumento da aplicação da pesquisa na realidade local, visto que as evidências coproduzidas são provavelmente mais realistas, aceitáveis e capazes de produzir mudanças mais duradouras (RUSHMER et al., 2019).

Observou-se que os profissionais da APS valoram o processo de territorialização que advém dos mapas, auxiliando no diagnóstico que a equipe faz do território. Os mapas são considerados ferramentas úteis para organizar, interpretar e comunicar os resultados para avaliação dos serviços de saúde. A elaboração e a interpretação de mapas pelas equipes consistem num processo de expressão gráfica, que considera o entendimento dos profissionais de saúde sobre seu território de atuação (GOLDSTEIN et al., 2013).

As evidências não são somente as evidências de resultados de pesquisas, coisas por exemplo, tabuladas, estudadas, pode ser um diagnóstico que a equipe faz do território [...] o que as agentes de saúde nos trazem [...]. Os mapas são evidências, os mapas que a gente fez (Rosa).

Entende-se que a identificação das características socioeconômicas, ambientais, bem como as potencialidades e prioridades da comunidade são considerados essenciais para a atuação sobre os determinantes da saúde e a implementação de estratégias de vigilância em saúde (MOYSÉS; FRANCO DE SÁ, 2014). Essas informaçôes podem ser captadas dando voz à comunidade, por intermédio das lideranças locais. Dessa 
maneira, para a operacionalização da evidência sociocultural, uma das ferramentas das equipes de ESF é o processo de territorialização, que resulta de uma pluralidade de informaçôes advindas tanto de dados epidemiológicos, quanto do mapeamento e de informantes chaves (líderes comunitários e conselheiros locais) (LIMA; GALIMBERTTI, 2016; FARIA, 2013).

Outro dispositivo para o reconhecimento da evidência sociocultural é a visita domiciliar, considerada uma tecnologia leve de trabalho, que permite, de forma privilegiada, conhecer o contexto familiar e social, bem como as relaçóes familiares, e como elas podem contribuir para o cuidado, cura ou recuperação daqueles que ali moram (SAVASSI, 2016; ANDRADE et al., 2014).

Ressalta-se que segundo a Portaria n. 2.436/2017 do Ministério da Saúde, é responsabilidade de todos profissionais das equipes que atuam na APS realizar visitas domiciliares, considerar as características sociais, econômicas, culturais, demográficas e epidemiológicas do território, para o planejamento local e a análise da situação de saúde, bem como implementar atividades de atenção à saúde de acordo com as necessidades da população, obedecendo a critérios de frequência, risco e vulnerabilidade, com base em parâmetros estabelecidos em evidências científicas.

Andermann e colaboradores (2016) asseguram que para ter um bom impacto na saúde em geral é importante, em primeiro lugar, entender quais são as verdadeiras necessidades de saúde para poder abordá-las de maneira informada por evidências. Uma avaliação objetiva das necessidades baseada em dados epidemiológicos, combinada com uma avaliaçáo subjetiva do que a comunidade pode considerar como as prioridades essenciais, é uma boa maneira de começar.

Em pesquisa realizada em Minas Gerais, Brasil, com o objetivo de analisar os atributos da APS, os autores concluíram que há necessidade de orientar as práticas de cuidado de acordo com os dados epidemiológicos e as necessidades da populaçáo, por meio da construção do diagnóstico local (GONTIJO et al., 2017).

Essa história de territorialização acho bem importante. Porque, por exemplo, nós tivemos a dengue, então nós tivemos cinco ou seis casos numa travessa [rua], então a gente se alertou de um jeito para aquele lugar que as agentes de saúde iam lá todos os dias, entâo isso eu acho importante, a localização é um dado, não deixa de ser uma evidência (Girassol).

Entender as preferências individuais e populacionais, bem como o contexto local, é primordial para escolher a melhor opção de qualquer implementação. Primeiramente, não adianta pensar em custos se a opção não for aceitável (ANDERMANN et al., 
2016). Outrossim, o relato de um profissional indica que na APS é essencial, em primeiro lugar, conhecer sua população adscrita, no seu âmbito individual e familiar, para depois aplicar uma evidência científica.

Tudo depende muito, vou citar um exemplo: tem uma senhora lá com os seus setenta anos e ela é hipertensa. Na consulta a pressão está "nas alturas", você vai olhar o tratamento dela tem três drogas otimizadas, mas por trás disso tem outras coisas, que são: problemas familiares, ela não sabe ler, tomar direito os remédios. Então, o que está no teu prontuário é o correto, mas não se sabe se ela tomou o remédio certo, se ela saber ler o receituário para tomar a medicação correta, não se sabe o que aconteceu na vida dela para a pressão estar assim. Então, na ESF é muito isso, é muito mais o teu primeiro feeling para depois você dar o segundo passo acadêmico, é saber a estrutura, o que aconteceu com ela. Se ela tem um filho que é drogado, presidiário, então a pressão vai estar sempre nas alturas, por mais que você otimize as drogas. Sempre a primeira coisa é o teu conhecimento em relação a tua área [território], as tuas famílias e depois você vai pensar clinicamente, depois você vai tratar, mas primeiro você tem que tentar entender (Gérbera).

Em suma, os profissionais da APS necessitam ser capazes de prover uma atenção integral, contínua e organizada à população adscrita, ou seja, devem ver o ser humano na sua complexidade, valorizando as relaçóes e compreendendo as pessoas nos seus contextos sociais (MINAYO, 2012). Diante disso, entende-se que para realizar um trabalho global, dos profissionais que atuam na APS, especificamente na ESF, é elementar que a PBE considere a evidência científica, a experiência clínica, a escolha do paciente e os aspectos socioculturais das pessoas/comunidades.

Nessa perspectiva, acredita-se que as informaçóes advindas da escuta atenta das necessidades da comunidade, do mapeamento que identifica áreas de risco e o perfil epidemiológico são evidências importantes a serem consideradas na prática dos profissionais (BREILH, 2015). Cabe à equipe da ESF identificar e valorizar as características socioculturais do território para uma PBS mais sensível à realidade e às necessidades da sua população adscrita. De fato, só porque uma intervenção funciona bem em um contexto não significa que ela será aplicável e transferível para outra.

Reconhece-se que integrar as evidências, valores e fatores contextuais para chegar a uma decisão final é, sem dúvidas, um desafio. É fundamental encontrar um equilíbrio, estimulando o diálogo entre as partes interessadas para prover decisões mais sutis, mais transparentes e, em última análise, mais propensas a ter impacto na melhoria da saúde (ANDERMANN et al., 2016). A figura 1 representa a proposta de inclusão do elemento "análise sociocultural" da PBE no âmbito da APS. 
Figura 1. Proposta dos Elementos da Prática Baseada em Evidência no Âmbito da Atençáo Primária à Saúde.

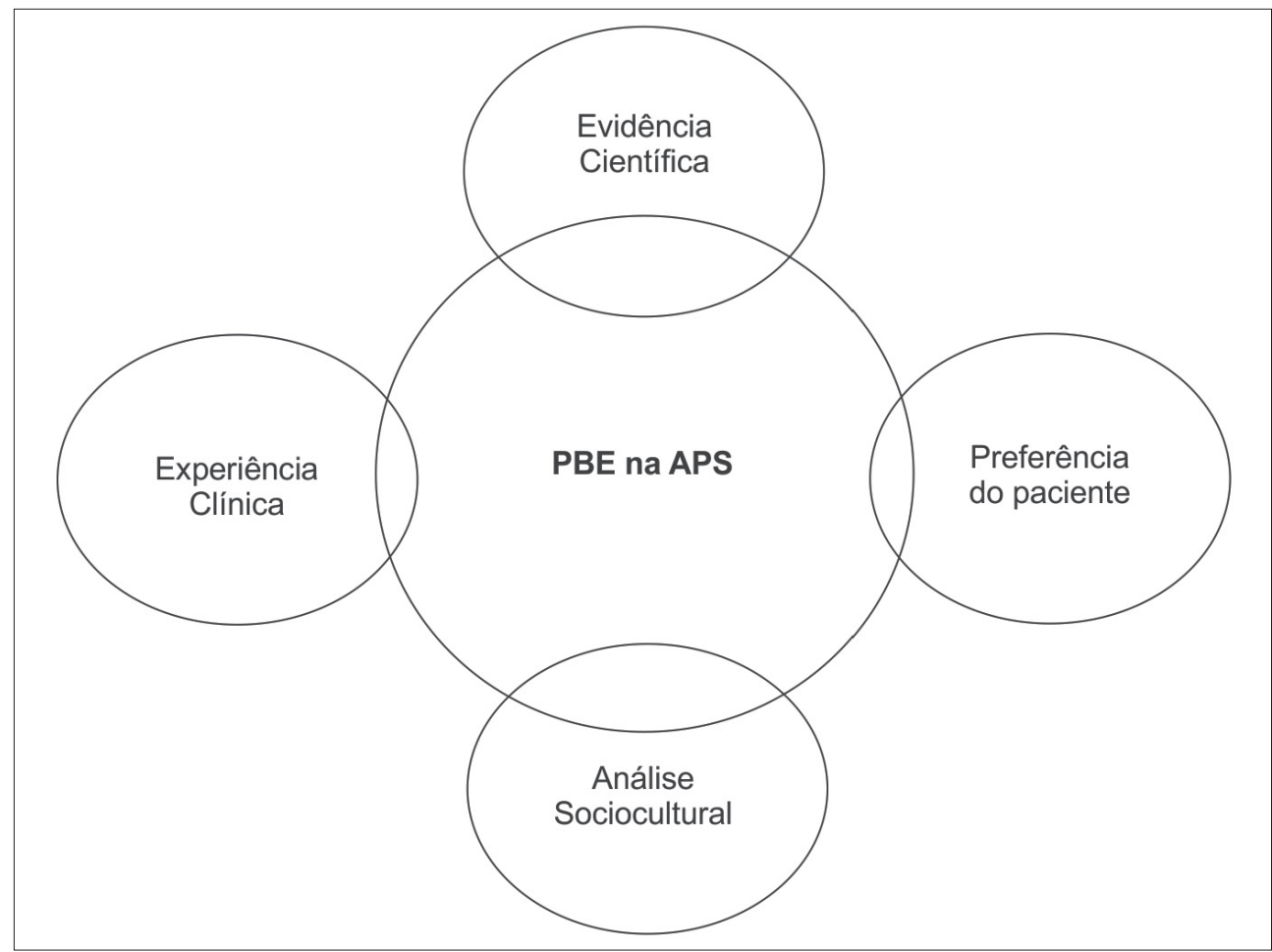

Fonte: Os autores.

\section{Considerações finais}

Identifica-se que os profissionais da ESF têm dificuldades em desenvolver uma prática em saúde voltada a evidências científicas. De acordo com os depoimentos, há uma deficiência de conhecimentos e habilidades para acessar fontes de informação (bases de periódicos), selecionar, interpretar e aplicar os resultados de pesquisas na prática da APS. Essa inabilidade, segundo os profissionais, advém do não exercício e aprendizagem da pesquisa científica na formação profissional, em nível de graduação e de educação continuada em serviço. Além disso, há um discurso contundente de que a alta demanda de trabalho na ESF limita o tempo para a busca e análise de estudos científicos. Por outro lado, a experiência profissional - individual e coletiva - é um aspecto valorizado pelos profissionais de saúde da ESF. 
Verifica-se, também que a PBE ainda está fortemente direcionada à assistência individual de atenção à saúde. Entretanto, o caráter coletivo da APS, por conseguinte, vai exigir que o profissional aplique uma pluralidade metodológica e de ferramentas para tomada de decisôes e resolutividade dos problemas de saúde nesse cenário. Nesse sentido, analisar os aspectos socioculturais que se dáo nos territórios permite compreender as determinaçóes sociais do processo saúde-doença. Portanto, para um profícuo desenvolvimento da PBE na APS, são oportunos métodos inovadores que considerem outras maneiras de ver e interpretar as situações de saúde-doença. Reconhecer os fenômenos socioculturais e ambientais do território, compreendendo a realidade num dado contexto, é uma forma de considerar as diversidades e o papel das variáveis sociais na vida dos indivíduos e coletividades no cuidado à saúde.

Vale ressaltar que não é propósito da pesquisa qualitativa a generalização dos resultados, mas percebe-se que ficou evidente a força dos aspectos socioculturais para uma abordagem mais realista e capaz de produzir mudanças significativas e duradouras nos serviços de atenção primária. ${ }^{1}$

\section{Agradecimentos}

À Coordenação de Aperfeiçoamento de Pessoal de Nível Superior (CAPES), pelo apoio financeiro por meio de bolsa de mestrado, e a todos os participantes do estudo.

\section{Referências}

AGREE Next Steps Consortium. The AGREE II Instrument. Canada, 2009. Disponível em: $<$ https://www.agreetrust.org/agree-ii/>

AMMOURI, A. et al. Evidence-Based Practice Knowledge, attitudes, practice and perceived barriers among nurses in Oman. Sultan Qaboos Univ Med. J., v. 14, n. 4, p. 537-45, 2014.

ANDERMANN, A. et al. Evidence for Health III: Making evidence- informed decisions that integrate values and context. Health Research Policy and Systems. v. 14, n. 16, 2016. Doi: 10.1186/ s12961-016-0085-4

ANDRADE, A. M. et al. Visita domiciliar: validação de um instrumento para registro e acompanhamento dos indivíduos e das famílias. Epidemiol. Serv. Saúde. Brasília, v. 23, n. 1, p. 165-175, jan-mar. 2014.

BAIRD, L. M. G.; MILLER, T. Factors influencing evidence-based practice for community nurses. British Journal of Community Nursing, v. 20, n. 5, 2015. 
BANDEIRA, A. G. et al. A utilização de um referencial metodológico na implementação de evidências como parte da investigação em enfermagem. Texto Contexto Enferm, v. 26, n. 4, p. e2550017, 2017.

BANNER, D. et al. Patient and Public Engagement in Integrated Knowledge Translation Research: Are we there yet? Research Involvement and Engagement. v. 5, n. 1, 2019. Doi: https:// doi.org/10.1186/s40900-019-0139-1

BARENDS, E et al. Managerial attitudes and perceived barriers regarding evidence-based practice: An international survey. PlosOne, v. 12, n. 10, p. e0184594, 2017. Doi: https://doi. org/10.1371/journal.pone.0184594.

BARDIN, L. Análise de conteúdo. Ed. rev. e ampl. São Paulo: Edições 70, 2016. 279 p.

BRASIL. Ministério da Saúde. Portaria no 2.436, de 21 de setembro de 2017. Aprova a Política Nacional de Atenção Básica, estabelecendo a revisão de diretrizes para a organização da Atenção Básica, no âmbito do Sistema Único de Saúde (SUS). Brasília, DF: Ministério da Saúde, 2017.

BREILH, J. Epidemiologia crítica ciência emancipadora e interculturalidade. 1 ed. 1 reimpr. Rio de Janeiro: Editora Fiocruz, 2015.

CAMARGO, F. C. et al. Avaliação de intervenção para difusão da enfermagem baseada em evidências em hospital de ensino. Rev Gaúcha Enferm., v. 37, n. esp., p. e68962, 2016.

COCHRANE BRASIL. Quem é e o que faz a Cochrane. Disponível em: http://brazil.cochrane. org/quem-\%C3\%A9-e-o-que-faz-cochrane. Acesso em: 4 ago. 2019.

EBELL, M. H. et al. How good is the evidence to support primary care practice? BMJ EvidenceBased Medicine. v. 22, p. 88-92, 2017.

FARIA, R.M. A Territorialização da Atenção Primária à Saúde no Sistema Único de Saúde e a construção de uma perspectiva de adequação dos serviços aos perfis do território. Hygeia v, 9, n. 16, p. 131-147, jun 2013.

GOLDSTEIN, R. et al. A experiência de mapeamento participativo para a construção de uma alternativa cartográfica para a ESF. Ciência \& Saúde Coletiva, v. 18, n. 1, p. 45-56, 2013.

GONTIJO, T. L. et al. Avaliação da atenção primária: o ponto de vista de usuários. Saúde debate, Rio de Janeiro, v. 41, n. 114, p. 741-752, jul-set. 2017.

INSTITUTO BRASILEIRO DE GEOGRAFIA E ESTATÍSTICA. Cidades. Disponível em: http://cidades.ibge.gov.br/xtras/perfil.php?codmun=420420. Acesso em: 4 ago. 2019.

KIRMAYER, L. Cultural competence and evidence-based practice in mental health: Epistemic communities and the politics of pluralism. Social Science \& Medicine, v. 75, p. 249-256, 2012. Doi: 10.1016/j.socscimed.2012.03.018 
LIMA, F. A.; GALIMBERTTI, P. A. Sentidos da participação social na saúde para lideranças comunitárias e profissionais da Estratégia Saúde da Família do território de Vila União, em Sobral-CE. Physis, Rio de Janeiro, v. 26, n. 1, p. 157-175, mar. 2016.

MALLION, J.; BROOKE, J. Community- and hospital-based nurses' implementation of evidence-based practice: are there any differences? British Journal of Community Nursing. v. 21, n. 3, p. 148-54, mar. 2016.

MINAYO, M. C. S. Saúde e Ambiente: uma relação necessária. In CAMPOS, G. W.S. et al. (Org.). Tratado de saúde coletiva. 2ed. rev. aum. São Paulo: Hucitec, 2012. p. 79-109.

MONKEN, B.; BARCELLOS, C. Vigilância em saúde e território utilizado: possibilidades teóricas e metodológicas. Cad. Saúde Pública, Rio de Janeiro, v. 21, n. 3. p. 898-906, maio-jun, 2005.

MONTENERY, S., et al. Cultural competence in nursing faculty: a journey, not a destination. Journal of Professional Nursing, v. 29, n. 6, p. 51-57, 2013. doi: dx.doi.org/10.1016/j. profnurs.2013.09.003

MORALES, C. F.; SANTACRUZ, C. J.; VERKOVITCH, I. Integración de la Enseñanza de la práctica de enfermería basada en la evidencia científica. Aquichán, Bogotá, v. 15, n. 4, p. 541553, dez. 2015.

MOYSÉS, S.T; FRANCO DE SÁ, R. Planos locais de promoção da saúde: intersetorialidade(s) construída(s) no território. Ciênc. saúde coletiva, Rio de Janeiro, v. 19, n. 11, p. 4323-4330, nov. 2014.

NAVABI, N. et al. Knowledge and Use of Evidence-based Dentistry among Iranian Dentists. Qaboos Univ Med J., v. 14, n. 2, p. 223-230, maio 2014.

PEREIRA, R. P. G. Enfermagem Baseada na Evidência: atitudes, barreiras e práticas. Tese (Doutorado) - Instituto de Ciências Biomédicas Abel Salazar da Universidade do Porto. Programa de Pós-Graduação em Ciências da Enfermagem, Porto, 2016.

PEREIRA, R. P. G.; CARDOSO, M. J. S.; MARTINS, M. A. C. Atitudes e barreiras à prática de enfermagem baseada na evidência em contexto comunitário. Rev. Enf. Ref., Coimbra. v. serIII, n. 7, p. 55-62, jul. 2012.

PERICAS-BELTRAN, J. et al. Perception of Spanish primary healthcare nurses about evidence based clinical practice: a qualitative study. Int. Nursing Rev, v.61, n. 1, p. 90-8, jan. 2014.

RUSHMER, R. et al. Knowledge translation: key concepts, terms and activities. In:

Population Health Monitoring: climbing the information pyramid. Switzerland: Springer International Publishing, 2019. doi: https://doi.org/10.1007/978-3-319-76562-4_7

SAVASSI, L. C. M. Os atuais desafios da Atenção Domiciliar na Atenção Primária à Saúde: uma análise na perspectiva do Sistema Único de Saúde. Rev Bras Med Fam Comunidade. Rio de Janeiro, v. 11, n. 38, p. 1-12, jan-dez, 2016. 
SACKETT, D. L. et al. Evidence based medicine: what it is and what it isn't. British Medical Journal, v.312, p. 71-72, jan. 1996.

. Medicina baseada em evidências: prática e ensino. 2 ed. Porto Alegre: Artmed, 2003.

SADEGHI-BAZARGAN, H.; TABRIZI, J.; AZAMI-AGHDASH, S. Barriers to evidencebased medicine: a systematic review. J Eval Clin Pract., v. 20, n. 6, p. 793-802, dez. 2014.

SANTOS, V. C.; ANJOS, K. F.; ALMEIDA, O. S. A percepção de formandos sobre a pesquisa em enfermagem no curso de graduação. Rev Enferm UFSM., v. 3, n. 1, p. 144-154, jan-abr. 2013. SAUNDERS, H.; VEHVILAINEN-JULKUNEN, K. Nurses' Evidence-Based Practice Beliefs and the Role of Evidence-Based Practice Mentors at University Hospitals in Finland. Worldviews on Evidence-Based Nursing. v. 14, n. 1, p. 35-45, 2017.

SHAFIEI, E. et al. Nurses' perceptions of evidence-based practice: a quantitative study at a teaching hospital in Iran. Med J Islam Repub Iran, v. 28, nov. 2014

SHIN, J.; LEE, E. The Influence of Social Capital on Nurse-Perceived Evidence-Based Practice Implementation in South Korea. J Nurs Scholarsh., v. 49, n. 3, p. 267-276, maio 2017.

SPERLING, S. Estratégia de Saúde da Família: a melhor aposta para um sistema de saúde orientado para a proteção de pessoas e conquistas civilizatórias. Reciis: Rev Eletron Comun Inf Inov Saúde, v. 11, n. 4, out-dez. 2017.

TAKEDA, S. A organização dos serviços de Atenção Primária à Saúde. In: DUNCAN, B. et al. (Orgs.). Medicina ambulatorial: condutas de atenção primária baseadas em evidências. 4. ed. Porto Alegre: Artmed, 2013. p. 19-32.

YODER, L. et al. Staff nurses' use of research to facilitate Evidence-Based Practice. Am J Nurs., v. 114, n. 9, Sept. 2014.

WERNECK, M. A. F.; FARIA, H. P.; CAMPOS, K. F.C. Protocolo de cuidados à saúde e de organizaçâo do serviço. Belo Horizonte: Nescon-UFMG; Coopmed, 2009. p. 13-49.

ZHOU, F. et al. Attitude, knowledge, and practice on Evidence-Based Nursing among registered nurses in traditional Chinese medicine hospitals: a multiple center cross-sectional survey in China. Evid Based Complement Alternat Med., ID 5478086, jun. 2016.

\section{Nota}

${ }^{1}$ L. R. Schneider participou da concepção, planejamento, análise, redação e revisão crítica do artigo. R. P. G. Pereira participou da análise, redação, revisão crítica e aprovação da versão final do artigo. L. Ferraz realizou a concepção, planejamento, análise, redação, revisão crítica e aprovação da versão final do artigo. 


\section{Abstract}

\section{Evidence-Based Practice and sociocultural analysis in Primary Care}

Evidence-Based Practice is an approach that has been renowned for improving clinical effectiveness and supporting health professional in their attitudes, by using three elements: scientific evidence, clinical experience and patients' preferences. This study aimed at understanding how Evidence-Based Practice in Primary Health Care is developed, specifically, referring to Family Health Strategy, as well as proposing the inclusion of the "socio-cultural analysis" element for decision making. It was a qualitative study, preceded by two focus groups, having the participation of 14 health professionals. The thematic content analysis technique was used to evaluate, handle and understand information. Family Health Strategy professionals have difficulties in developing health practice focused on scientific evidence, showing their lack of knowledge and skills for research and also heavy workload.

Moreover, results showed that Evidence-Based Practice should be differentiated according to the reality of the places where health professional teams work. It is the responsibility of health professionals to identify and to evaluate the sociocultural characteristics of the community for an Evidence-Based Practice that is more sensitive to the health needs of the population involved.

> Keywords: Evidence-Based Practice; Primary Health Care; Family Health Strategy; cultural characteristics. 